\title{
QUEEN'S
UNIVERSITY
BELFAST
}

\section{Towards Holographic Beam-Forming Metasurface Technology for Next Generation CubeSats}

\author{
Yurduseven, O., Podilchak, S., \& Khalily, M. (2020). Towards Holographic Beam-Forming Metasurface \\ Technology for Next Generation CubeSats. In 2020 International Conference on UK-China Emerging \\ Technologies (UCET) https://doi.org/10.1109/UCET51115.2020.9205418
}

Published in:

2020 International Conference on UK-China Emerging Technologies (UCET)

\section{Document Version:}

Peer reviewed version

Queen's University Belfast - Research Portal:

Link to publication record in Queen's University Belfast Research Portal

Publisher rights

Copyright 2020 IEEE. This work is made available online in accordance with the publisher's policies. Please refer to any applicable terms of use of the publisher.

\section{General rights}

Copyright for the publications made accessible via the Queen's University Belfast Research Portal is retained by the author(s) and / or other copyright owners and it is a condition of accessing these publications that users recognise and abide by the legal requirements associated with these rights.

Take down policy

The Research Portal is Queen's institutional repository that provides access to Queen's research output. Every effort has been made to ensure that content in the Research Portal does not infringe any person's rights, or applicable UK laws. If you discover content in the Research Portal that you believe breaches copyright or violates any law, please contact openaccess@qub.ac.uk. 


\section{Towards Holographic Beam-Forming Metasurface Technology for Next Generation CubeSats}

\author{
Okan Yurduseven \\ Centre for Wireless Innovation \\ Queen's University Belfast \\ Belfast, Northern Ireland, UK \\ okan.yurduseven@qub.ac.uk
}

\author{
Symon Podilchak \\ School of Engineering \\ University of Edinburgh \\ Edinburgh, Scotland, UK \\ S.Podilchak@ed.ac.uk
}

\author{
Mohsen Khalily \\ Institute for Communication Systems \\ University of Surrey \\ Surrey, England, UK \\ m.khalily@surrey.ac.uk
}

\begin{abstract}
We present the concept of holographic beam forming metasurface antenna for CubeSat platforms at $\mathrm{X}$-band frequencies. The proposed metasurface topology exhibits a flat-panel system layout, particularly desirable for integration with CubeSat platforms without a hardware-intense deployment mechanism for launch. It is shown that appropriately interacting the guidedmode (or reference-wave) with a metasurface layer makes it possible to realize the radiation pattern of interest as an objective function - similar to an optical hologram - without the need for dedicated phase shifting circuits. The full-wave simulations of the designed metasurface layer integrated with a $1 \mathrm{U}$ CubeSat reveals a high-fidelity beam-control with an aperture efficiency greater than $40 \%$ at $10 \mathrm{GHz}$ operating frequency.
\end{abstract}

Index Terms-metasurface, holography, CubeSat, beamforming, microwaves.

\section{INTRODUCTION}

Metamaterials are artificial, sub-wavelength structures enabling electromagnetic (EM) properties that might not be achievable from natural materials [1]-[3]. Planar surfaces synthesized using an array of sub-wavelength metamaterial elements (or meta-elements) are known as metasurfaces [4], [5]. An interesting property of metasurfaces is that they can be engineered to synthesize an arbitrary wavefront in an allelectronic manner. Beam-synthesis is an important application of EM wave-control and has been studied extensively within the field of antennas and propagation, particularly in the context of phased arrays [6]-[8]. Conventionally, beam-synthesis requires an array topology, in which an array of antennas, typically sampled at a Nyquist limit, is used. This technique, known as phased arrays, requires that each antenna within the synthesized array aperture is connected to a dedicated phase-shifting circuit to realize the desired phase profile across the array aperture. Moreover, due to the high insertion loss characteristics of these phase shifting circuits, power amplifiers are also needed to compensate for their insertion losses. As a result, the conventional phase shifting technology can be power hungry and exhibit a significant complexity in the hardware layer. Metasurfaces offer an alternative solution to the beam-synthesis problem and, as will be shown in this paper, can be used to break the conventional phase shifting requirements of the phased array technology to achieve beam

The work of O. Yurduseven was in part supported by a research grant from the Leverhulme under Research Leadership Award RL-2019-019. forming, significantly simplifying the hardware architecture and power consumption metrics. This advantage of metasurfaces makes them a suitable candidate for Earth and space science applications. Instruments for these missions typically exhibit challenging payload requirements, such as limited space available for antenna integration and tight component weight tolerances to name a few. Moreover, because these applications require establishing communication links over extremely large distances, high gain antennas are needed to satisfy the required link budgets. The high gain requirement, in turn, increases the size of the antenna to achieve the desired gain metrics, which further complicates the deployment process. A typical example for such kind of antenna is the meshed reflector topology, which was successfully demonstrated for several missions, including NASA's Ka-band parabolic Deployable Antenna (KapDA) [9] and deployable Ka-band anTENna (KaTENna) [10], and ESA ARTES project Reflective Metal Mesh (MESNET) and European H2020 project Large European Antenna (LEA) [11], [12]. The deployment of meshed reflector antennas in space has been proven to be an extremely challenging task for these applications. Because of the limited space available, systems leveraging these antennas require a special deployment mechanism in which the reflector antennas are folded prior to launch and unfolded once the instrument is deployed. This approach brings several disadvantages. First, the weight of the deployment mechanism substantially adds to the total weight budget allocated for the antenna platform within the instrument. Second, the necessity to have such a deployment mechanism can substantially increase the cost of the mission due to the precise alignment requirements of the different parts of the antenna once it is launched and unfolded. To address these challenges, the concept of flat-panel antennas has recently gained substantial traction. A good example for this is NASA's MarCO mission, in which the communication link is established using a flat-panel reflectarray antenna topology [13]. Due to its flat-panel geometry, the deployment mechanism of this antenna has been shown to be simpler while the antenna offers on par gain characteristics in comparison to the meshed reflector architecture. Although the reflectarray surface itself has a flat-panel topology, conventional reflectarray antennas require a secondary feed to illuminate the reflectarray surface. This requirement makes reflectarray 
antennas not truly flat-panel antennas but rather volumetric structures. Recently, the concept of flat-panel, holographic metasurface antennas has received significant interest from the antenna community, particularly due to their advantage to solve these issues. A flat-panel metasurface antenna uses an integrated feed architecture, which lies on the same plane as the metasurface, resulting in a truly flat system layout. The design approach of such an antenna relies on a holographic principle, using the electromagnetic (EM) waves launched by its feed as a reference-wave. Interacting the metasurface with the reference-wave produces the desired objective function (or aperture field distribution), similar to a hologram at optical frequencies. In Section II, we demonstrate the concept of holographic metasurface antennas as applied to next generation CubeSat technologies.

\section{Holographic Metasurface Design}

Holography is a well-known concept at optical frequencies and there has been a substantial amount of research conducted in this field since the revolutionary works of Gabor [14] and Upatnieks [15]. The application of holography has recently gained traction in a variety of applications at microwave and millimetre-wave frequencies, such as radar imaging [16]-[20], compressive sensing [21]-[26], antenna characterization [27][30] and beam-synthesis [31]-[34] to name a few. Leveraging the holographic concept to achieve beam-synthesis at microwave and millimetre-frequencies requires that a referencewave is synthesized at these frequencies which can be modulated in such a way that the resultant wavefront produces the radiation pattern of interest in the far-field of the antenna. This modulation step can be realized using a metasurface layer, which, when illuminated by the reference-wave, produces the desired radiation wavefront, similar to an optical hologram. This simple yet extremely powerful concept does not require any phase shifting circuits to achieve beam-forming, and therefore, can exhibit a significantly simplified system architecture on the physical layer. A technique to synthesize a desired radiated wave-front using a metasurface aperture is known as discrete surface impedance, approximating a smooth and continuous impedance function. This method has been well reported in the literature with numerous metasurface antenna prototypes are now presented [35]-[38]. Conventionally, this technique requires creating an impedance database by means of eigenmode simulations of a single metamaterial element with varying dimensions and/or geometries. Different from the holographic principle relying on a discrete dipole mechanism to model [33], creating a continuous impedance function requires that a smoothly varying surface impedance profile is achieved. This constraint is not always feasible to synthesize different wavefronts to realize different radiation patterns. In the presented holographic principle, we achieve beam-forming by directly interacting the array factor (AF) of the sub-wavelength sampled metasurface aperture and the holographic guided-mode reference-wave by relying on a polarizable dipole model for the meta-elements building the metasurface. This enables us to modulate and synthesize the desired antenna wavefront by employing discontinuously varying elements and simple modulation schemes, such as binary (on/off) modulation. Such modulation schemes can be realized using simple, semiconductor circuit components, such as PIN diodes [32]. A metasurface is synthesized using an array of meta-elements distributed across a planar aperture. Hence, it can be said that a metasurface antenna consists of an array of sub-wavelength sized meta-elements. Moreover, a metasurface aperture is sampled at a sub-wavelength level, meaning that the spacing between the meta-elements is subwavelength. In order to illustrate the metasurface design and the holographic beam-forming technique, let us consider the 2D aperture shown in Fig. 1.

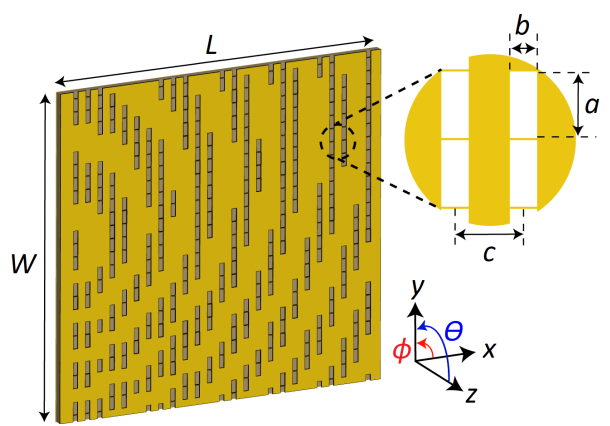

(a)

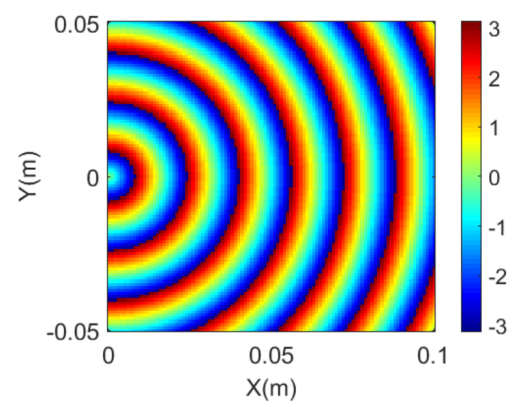

(b)

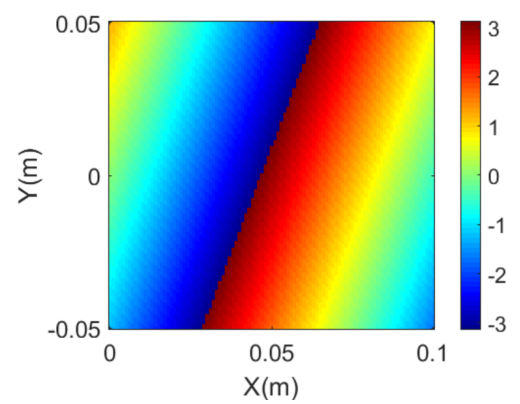

(c)

Fig. 1: Flat-panel metasurface antenna (a) structure of the metasurface antenna (b) example guided-mode phase pattern (c) example objective function phase pattern to be realized. Dimensions: $a=3.88 \mathrm{~mm}, b=1.6 \mathrm{~mm}, c=4 \mathrm{~mm}, W=L=10$ $\mathrm{cm}$. Objective function is selected to be $\left(\theta=20^{\circ}, \phi=20^{\circ}\right)$ for depiction purposes only. Phase patterns are in radians.

For the presented example in Fig. 1, the metasurface antenna 
consists of a parallel plate waveguide (PPW) layout, with a dielectric substrate (Rogers 4003, $\epsilon_{r}=3.38$ and $\tan \delta=0.0027$ ), in between a ground plane at the bottom and a metasurface layer on top. For this design, the frequency band is selected to be $10 \mathrm{GHz}$ within the X-band. In Fig. 1, the metasurface layer is synthesized using an array of sub-wavelength sized slot-shaped meta-elements. The metasurface is fed through a coaxial cable exciting a waveguide to launch a holographic reference-wave into the dielectric substrate. The launched reference-wave within the PPW has a quasi-TEM mode characteristic and its magnetic field can be modeled as follows:

$$
\mathbf{H}_{a, b}=H_{0}^{1}\left(k_{g} \mathbf{r}_{a, b}\right) \sin \left(\zeta_{a, b}\right)
$$

In (1), $\mathbf{H}$ is the magnetic field component launched into the dielectric substrate, $H_{0}^{1}$ is the Hankel function that models an outgoing cylindrical wave, $k_{g}$ is the wavenumber in dielectric, $\mathbf{r}$ is the distance vector for each meta-element from the feed point, and $\zeta$ is the angle of the distance vector $\mathbf{r}$ with respect to the $\mathrm{x}$-axis while $a$ and $b$ denote the index numbers for the meta-elements across the metasurface as follows:

$$
\mathbf{r}_{a, b}=\sqrt{\left(x_{a}-x_{f}\right)^{2}+\left(y_{b}-y_{f}\right)^{2}}
$$

For the metasurface layout depicted in Fig. 1, in (2), the coordinates of the feed point is $\left(x_{f}=0, y_{f}=0\right)$. Here, we focus our attention to the magnetic field component due to the slot geometry of the meta-elements, exhibiting magnetic dipole characteristics [33]. It should be noted that, due to their large aspect ratio, the meta-elements are linearly polarized along the $\mathrm{y}$-axis, and hence, we reduce the design problem at hand to a scalar problem by considering only the y-polarized magnetic field introduced by the sinusoidal term in (2). Despite the scalar approximation, we use the bold font to denote the vector - matrix notation. Considering that the meta-elements couple to the guided-mode and radiate into free space, the presented metasurface architecture of Fig. 1 is a transmission hologram, radiating in direction $(\theta, \phi)$ in the far-field with the array factor (AF) of the aperture can be defined as follows:

$$
\begin{aligned}
A F(\theta, \phi)= & \sum_{a=1}^{N} \sum_{b=1}^{M} H_{0}^{1}\left(k_{g} \mathbf{r}_{a, b}\right) \sin \left(\zeta_{a, b}\right) \\
& e^{-j k_{0} x_{a} \sin (\theta) \cos (\phi)} e^{-j k_{0} y_{b} \sin (\theta) \sin (\phi)}
\end{aligned}
$$

From (3), it is evident that, to steer the beam from such an aperture in a given $(\theta, \phi)$ direction, the exponent of the exponential term needs to equal zero. From the AF projection in (3), the phase at each meta-element position can be given as follows:

$$
\begin{array}{r}
\psi(a, b)=\angle H_{0}^{1}\left(k_{g} \mathbf{r}_{a, b}\right) \sin \left(\zeta_{a, b}\right) e^{-j k_{0} x_{a} \sin (\theta) \cos (\phi)} \\
e^{-j k_{0} y_{b} \sin (\theta) \sin (\phi)}
\end{array}
$$

In order to maximize the AF of (3) in a given direction $(\theta, \phi)$, we define a phase grating with a phase conjugate of (3), $\psi^{*}(a, b)$. Following this selection, we activate only the meta-elements where remains below a certain threshold, which was found to be $\pm 50^{\circ}$ as a result of a parametric analysis. The etched meta-atoms shown in Fig. 1 are the meta-atoms at which the phase error is below the selected threshold.

\section{CubeSat Integrated Metasurface Antenna AND X-BAND BEAM FORMING}

Leveraging the holographic beam-synthesis procedure described in (1)-(4), we design a flat-panel metasurface antenna for integration with a $1 \mathrm{U}$ CubeSat platform $(10 \mathrm{~cm} \times 10 \mathrm{~cm}$ $\mathrm{x} 10 \mathrm{~cm}$ ) at $10 \mathrm{GHz}$ frequency (X-band). The size of the metasurface is $10 \mathrm{~cm} \times 10 \mathrm{~cm}$ and is mounted onto the front surface of the CubeSat as depicted in Fig. 2.

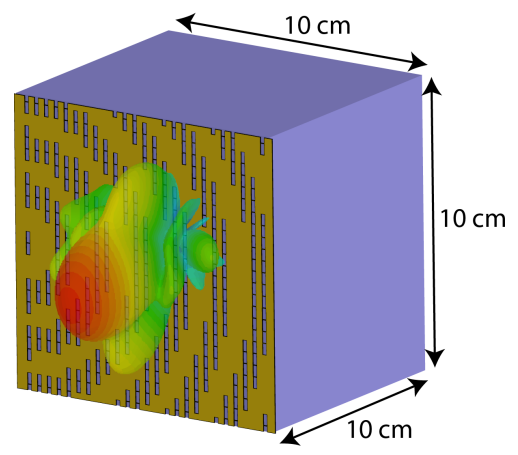

Fig. 2: Depiction of the X-band metasurface antenna radiating in the broadside direction integrated with a CubeSat platform.

The metasurface antenna is designed to radiate in the broadside direction $\left(\theta=0^{\circ}, \phi=0^{\circ}\right)$. Therefore, the objective function for the design process is the projection of a plane wave with a uniform phase across the metasurface aperture. Although shown for broadside radiation, the same holographic modulation principle can be followed to steer the beam in any arbitrary direction. The design process of the metasurface antenna consists of two steps. First, we use the analytical model of (1)-(4) to calculate the metasurface layer. Second, the designed metasurface layer is imported into a full-wave EM simulation software, CST Microwave Studio, to carry out the radiation analysis of the calculated metasurface layer. The radiation pattern of the metasurface antenna is shown in Fig. 3. For this demonstration, the E-plane is $\left(\phi=0^{\circ}, \theta=0^{\circ} \rightarrow 90^{\circ}\right)$ while the H-plane is $\left(\phi=90^{\circ}, \theta=0^{\circ} \rightarrow 90^{\circ}\right)$.

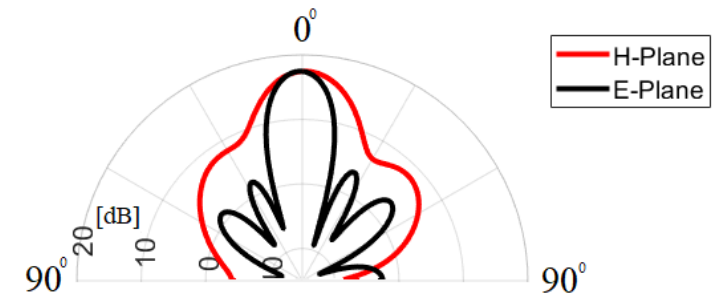

Fig. 3: Radiation patterns of the X-band metasurface antenna. 
The sidelobe levels are recorded to be $-10 \mathrm{~dB}$ for the $\mathrm{H}$-plane and $-13.8 \mathrm{~dB}$ for the E-plane, respectively. The directivity of the metasurface is $17.5 \mathrm{dBi}$, resulting in an aperture efficiency of $41 \%$.

\section{CONCLUSION}

We have presented a holographic beam-forming metasurface antenna for integration with next generation CubeSat platforms at microwave frequencies. The full-wave simulations of the CubeSat integrated metasurface concept have shown that the metasurface antenna can achieve high-fidelity beam-forming using a truly flat-panel hardware architecture in a holographic manner, eliminating need for hardware intense and powerhungry phase-shifting circuits.

\section{REFERENCES}

[1] N. Engheta and R. W. Ziolkowski, Metamaterials: physics and engineering explorations. John Wiley \& Sons, 2006.

[2] V. M. Shalaev, W. Cai, U. K. Chettiar, H.-K. Yuan, A. K. Sarychev, V. P. Drachev, and A. V. Kildishev, "Negative index of refraction in optical metamaterials," Optics letters, vol. 30, no. 24, pp. 3356-3358, 2005.

[3] D. Schurig, J. J. Mock, B. Justice, S. A. Cummer, J. B. Pendry, A. F. Starr, and D. R. Smith, "Metamaterial electromagnetic cloak at microwave frequencies," Science, vol. 314, no. 5801, pp. 977-980, 2006.

[4] S. B. Glybovski, S. A. Tretyakov, P. A. Belov, Y. S. Kivshar, and C. R. Simovski, "Metasurfaces: From microwaves to visible," Physics reports, vol. 634, pp. 1-72, 2016.

[5] S. Maci, G. Minatti, M. Casaletti, and M. Bosiljevac, "Metasurfing: Addressing waves on impenetrable metasurfaces," IEEE Antennas and Wireless Propagation Letters, vol. 10, pp. 1499-1502, 2011.

[6] G. Mishra, S. K. Sharma, J.-C. S. Chieh, and J. Rowland, "W-band circular polarized series fed single plane beamsteering array antenna with 4-bit phase shifter for cubesat applications," in 2017 IEEE International Symposium on Antennas and Propagation \& USNC/URSI National Radio Science Meeting, pp. 2555-2556, IEEE, 2017.

[7] K. LaCalli, "Phased helical antenna array design for cubesat application," in 2018 IEEE Aerospace Conference, pp. 1-13, IEEE, 2018.

[8] J. Long, D. Thorsen, and O. Kegege, "Retrodirective phased array antenna for cubesats," in 2019 IEEE Aerospace Conference, pp. 1-11, IEEE, 2019

[9] N. Chahat, R. E. Hodges, J. Sauder, M. Thomson, and Y. Rahmat-Samii, "The deep-space network telecommunication cubesat antenna: Using the deployable ka-band mesh reflector antenna.," IEEE Antennas and Propagation Magazine, vol. 59, no. 2, pp. 31-38, 2017.

[10] N. Chahat, J. Sauder, M. Mitchell, N. Beidleman, and G. Freebury, "One-meter deployable mesh reflector for deep space network telecommunication at $\mathrm{x}$-and ka-band," IEEE Transactions on Antennas and Propagation, 2019.

[11] L. Datashvili, N. Maghaldadze, and L. Dufour, "Structural solutions of deployable antennas for small satellites," in 2019 13th European Conference on Antennas and Propagation (EuCAP), pp. 1-4, IEEE, 2019.

[12] L. Datashvili, N. Maghaldadze, H. Baier, M. Friemel, and L. da RochaSchmidt, "High trl achieved for european large deployable reflector antennas," 2018

[13] R. E. Hodges, N. Chahat, D. J. Hoppe, and J. D. Vacchione, "A deployable high-gain antenna bound for mars: Developing a new foldedpanel reflectarray for the first cubesat mission to mars.," IEEE Antennas and Propagation Magazine, vol. 59, no. 2, pp. 39-49, 2017.

[14] D. Gabor, "A new microscopic principle," 1948.

[15] E. N. Leith and J. Upatnieks, "Reconstructed wavefronts and communication theory," JOSA, vol. 52, no. 10, pp. 1123-1130, 1962.

[16] D. Smith, O. Yurduseven, B. Livingstone, and V. Schejbal, "Microwave imaging using indirect holographic techniques," IEEE Antennas and Propagation Magazine, vol. 56, no. 1, pp. 104-117, 2014.

[17] L. Wang, "Enhanced holographic microwave imaging for mnp target tumor detection," IEEE Access, vol. 7, pp. 37167-37180, 2019.
[18] R. K. Amineh, M. Ravan, J. McCombe, and N. K. Nikolova, "Threedimensional microwave holographic imaging employing forwardscattered waves only," International Journal of Antennas and Propagation, vol. 2013, 2013.

[19] O. Yurduseven, "Indirect microwave holographic imaging of concealed ordnance for airport security imaging systems," Progress in Electromagnetics Research, vol. 146, pp. 7-13, 2014.

[20] D. Sheen, D. McMakin, and T. Hall, "Near-field three-dimensional radar imaging techniques and applications," Applied Optics, vol. 49, no. 19, pp. E83-E93, 2010.

[21] O. Yurduseven, D. L. Marks, T. Fromenteze, J. N. Gollub, and D. R. Smith, "Millimeter-wave spotlight imager using dynamic holographic metasurface antennas," Optics Express, vol. 25, no. 15, pp. 18230$18249,2017$.

[22] J. Hunt, T. Driscoll, A. Mrozack, G. Lipworth, M. Reynolds, D. Brady, and D. R. Smith, "Metamaterial apertures for computational imaging," Science, vol. 339, no. 6117, pp. 310-313, 2013.

[23] O. Yurduseven, V. R. Gowda, J. N. Gollub, and D. R. Smith, "Printed aperiodic cavity for computational and microwave imaging," IEEE Microwave and Wireless Components Letters, vol. 26, no. 5, pp. 367369, 2016.

[24] T. Sleasman, M. F. Imani, J. N. Gollub, and D. R. Smith, "Dynamic metamaterial aperture for microwave imaging," Applied Physics Letters, vol. 107, no. 20, p. 204104, 2015.

[25] O. Yurduseven, J. N. Gollub, D. L. Marks, and D. R. Smith, "Frequencydiverse microwave imaging using planar mills-cross cavity apertures," Optics express, vol. 24, no. 8, pp. 8907-8925, 2016.

[26] T. Zvolensky, V. R. Gowda, J. Gollub, D. L. Marks, and D. R. Smith, "W-band sparse imaging system using frequency diverse cavity-fed metasurface antennas," IEEE Access, vol. 6, pp. 73659-73668, 2018.

[27] D. Smith, S. P. Skobelev, and M. P. Leach, "A modified holographic technique for antenna measurements," in 2008 8th International Symposium on Antennas, Propagation and EM Theory, pp. 46-49, IEEE, 2008.

[28] A. Arboleya, J. Laviada, J. Ala-Laurinaho, Y. Álvarez, F. Las-Heras, A. V. Räisänen, et al., "Indirect off-axis holography for antenna metrology," Holographic Materials and Optical Systems, p. 243, 2017.

[29] E. B. Joy, "Microwave holography for antenna and radome diagnostics," in IEEE Antennas and Propagation Society International Symposium. 2001 Digest. Held in conjunction with: USNC/URSI National Radio Science Meeting (Cat. No. 01CH37229), vol. 4, pp. 440-443, IEEE, 2001.

[30] A. Arboleya, J. Ala-Laurinaho, J. Laviada, Y. Álvarez, F. Las-Heras, and A. V. Räisänen, "Millimeter-wave phaseless antenna measurement based on a modified off-axis holography setup," Journal of Infrared, Millimeter, and Terahertz Waves, vol. 37, no. 2, pp. 160-174, 2016.

[31] O. Yurduseven and D. R. Smith, "Dual-polarization printed holographic multibeam metasurface antenna," IEEE Antennas and Wireless Propagation Letters, vol. 16, pp. 2738-2741, 2017.

[32] O. Yurduseven, D. L. Marks, T. Fromenteze, and D. R. Smith, "Dynamically reconfigurable holographic metasurface aperture for a millscross monochromatic microwave camera," Optics express, vol. 26, no. 5, pp. 5281-5291, 2018

[33] D. R. Smith, O. Yurduseven, L. P. Mancera, P. Bowen, and N. B. Kundtz, "Analysis of a waveguide-fed metasurface antenna," Physical Review Applied, vol. 8, no. 5, p. 054048, 2017.

[34] A. Araghi, M. Khalily, P. Xiao, and R. Tafazolli, "Holographic-based mmw-wideband bidirectional frequency scanning leaky wave antenna," EUCAP 2020, 2020.

[35] B. H. Fong, J. S. Colburn, J. J. Ottusch, J. L. Visher, and D. F. Sievenpiper, "Scalar and tensor holographic artificial impedance surfaces," IEEE Transactions on Antennas and Propagation, vol. 58, no. 10, pp. 3212-3221, 2010.

[36] G. Minatti, F. Caminita, M. Casaletti, and S. Maci, "Spiral leaky-wave antennas based on modulated surface impedance," IEEE Transactions on antennas and propagation, vol. 59, no. 12, pp. 4436-4444, 2011.

[37] S. Pandi, C. A. Balanis, and C. R. Birtcher, "Design of scalar impedance holographic metasurfaces for antenna beam formation with desired polarization," IEEE Transactions on Antennas and Propagation, vol. 63, no. 7, pp. 3016-3024, 2015.

[38] M. Faenzi, G. Minatti, D. Gonzalez-Ovejero, F. Caminita, E. Martini, C. Della Giovampaola, and S. Maci, "Metasurface antennas: New models, applications and realizations," Scientific reports, vol. 9, no. 1, pp. 1-14, 2019. 